\title{
Les Fils de Bas-de-Cuir. Sauvagisme, franc trappeur et « lutte des traces » dans le roman d'aventures français (1850-1880)
}

\section{Luca Di Gregorio}

\section{OpenEdition}

Journals

\section{Édition électronique}

URL : https://journals.openedition.org/transatlantica/10688

DOI : $10.4000 /$ transatlantica. 10688

ISSN : $1765-2766$

\section{Éditeur}

Association française d'Etudes Américaines (AFEA)

\section{Référence électronique}

Luca Di Gregorio, « Les Fils de Bas-de-Cuir. Sauvagisme, franc trappeur et «lutte des traces » dans le roman d'aventures français (1850-1880) », Transatlantica [En ligne], 2 | 2017, mis en ligne le 13 mai 2019, consulté le 02 février 2023. URL : http://journals.openedition.org/transatlantica/10688 ; DOI : https://doi.org/10.4000/transatlantica.10688

Ce document a été généré automatiquement le 2 février 2023.

\section{c) (†)}

Creative Commons - Attribution - Pas d'Utilisation Commerciale - Pas de Modification 4.0 International - CC BY-NC-ND 4.0

https://creativecommons.org/licenses/by-nc-nd/4.0/ 


\title{
Les Fils de Bas-de-Cuir. Sauvagisme, franc trappeur et « lutte des traces » dans le roman d'aventures français (1850-1880)
}

\author{
Luca Di Gregorio
}

\section{Introduction}

1 En 1853, Gabriel Ferry publie Le Coureur des bois. Ce roman-feuilleton, largement inspiré du cycle de Bas-de-Cuir de James Fenimore Cooper, est parfois considéré comme «le premier western français " (Mathé 185; Wolff 143). D’autres étiquettes ont également pu lui être accolées, insistant davantage sur sa composante amérindienne: "roman mohican ", «roman d'Indiens", «roman de Sauvages", "roman d'aventures américaines ", etc. (Billington; Bleton). Les années 1860-1880 sont ensuite marquées par l'abondante production de Gustave Aimard, qui fera du personnage du coureur des bois (ou franc trappeur) l'un des grands héros populaires français du xix siècle. Ce sillon aventureux sera enfin prolongé, plus ou moins épisodiquement, par des épigones d'Aimard, non seulement en France (Louis Noir, Louis Boussenard) mais aussi à l'étranger (par exemple l'Italien Emilio Salgari). Au Xx siècle, la mémoire du coureur des bois, précisément irriguée par cette littérature populaire, continuera d'être évoquée par des auteurs aussi différents que Robert Desnos, Maurice Genevoix ou Michel Le Bris - soit en souvenir de leurs lectures d'enfance, soit pour renouer, à l'heure où triomphe le western, avec un mode d'habitation français de l'Amérique.

2 L'indécision qui s'est perpétuée, du XIX siècle à nos jours, quant à l'étiquette à attribuer au roman de l'Ouest pratiqué par Ferry, Aimard et leurs épigones, semble sans équivalent pour les autres sous-genres aventureux du XIXe siècle, à propos desquels, peu ou prou, chacun s'accorde désormais ${ }^{1}$. Pareille hésitation trahit un malentendu persistant quant aux rapports spécifiques (de rupture médiatique? de continuité 
culturelle ?) qu'entretient cette forme d'aventure dans l'Ouest avec la formule que les États-Unis ont imposée à travers le western, genre tout droit issu du mythe pionnier de la Frontière. Il y aurait lieu de se demander, indépendamment des circuits médiatiques véhiculant les récits, dans quelle mesure coureur des bois et pionnier peuvent se recouper ou, au contraire, diverger dans leurs aventures respectives. En faisant valoir qu'au XIX ${ }^{e}$ siècle, les ruptures entre belles-lettres et régimes de production populaires n'excluent pas certaines continuités culturelles et imaginaires, nous analyserons le genre aventureux mettant en scène ce coureur des bois - genre que l'on appellera roman sauvagiste - comme un genre qui emprunte à l'intergénéricité du roman populaire français pour rendre romanesque un imaginaire assez diffus de l'Amérique, remontant aux projections discursives héritées des Lumières sur «le Sauvage». Ce substrat de textes et d'images imprime au roman d'Aimard un aventurisme singulier, irréductible à l'imaginaire "d'effraction» qu'est le western, mais aussi aux dépaysements exotiques du roman colonial.

3 Dans le sillage de travaux déjà publiés (Di Gregorio 2014, 2017, 2018), cet article se situe dans le prolongement d'une série études ayant ravivé l'intérêt de la recherche, depuis le début des années 2000, pour la figure de Gustave Aimard et le genre du roman sauvagiste. En particulier, nous voudrions apporter quelques précisions supplémentaires sur l'importance de ce corpus pour l'histoire française de l'imagination de l'Amérique. Ces dernières années, en effet, deux perspectives dominantes semblent s'être partagé le traitement de cette matière romanesque. La première, portée par des historiens comme Tangi Villerbu (2007), a mis en lumière un certain nombre de regrets historiques que les Français, à travers des auteurs comme Aimard, ont voulu opposer au peuplement accéléré de l'Ouest par les Américains. À michemin entre le déni et le contrepied historiographique, le roman du coureur des bois tracerait ainsi un « récit parallèle » ou une « vision » alternative à cette conquête, sorte de négatif idéal par lequel une France mise hors-jeu du Nouveau Monde voudrait contrebattre la geste pionnière. Malgré son relatif désintérêt pour les spécificités propres à la fiction, cette perspective, au fond assez proche de l'imagologie dont s'occupe aussi la littérature comparée, pointe très justement l'existence d'une volonté en France de fixer une représentation de l'Ouest antérieure à l'épopée pionnière.

4 La seconde perspective, si elle interroge plus directement le fait romanesque, met avant tout l'accent sur le système de genres, de supports et de signes médiatiques dans lequel s'inscrit la production des romans sauvagistes entre 1850 et 1880. Matthieu Letourneux (2010) a bien établi que le roman sauvagiste, parce qu'il ne se sépare pas de la forme roman-feuilleton, construit ses codes aventureux à l'intersection de plusieurs sous-genres, mais aussi à l'intérieur de circuits médiatiques qui ne concernent pas seulement ni directement les thèmes américains. Il nous semble qu'il y aurait lieu cependant d'entendre de façon plus «dialectique » la valeur explicative donnée à ces phénomènes d'étiquetage et de circulation médiatiques dans le contexte de la première culture de masse. Ces critères tendent en effet à considérer la culture populairemédiatique comme un corpus autarcique, régi tout entier par des programmes internes de production, de thématisation et de narrativité. Si elle était exclusive, cette phénoménologie médiatique courrait le risque d'estomper, et parfois de réduire à l'os, la pertinence et la persistance culturelle que conservent certains imaginaires même en régime médiatique. En effet, y compris lorsqu'ils se cristallisent dans des formes contraintes par le format, la collection ou toute autre forme de sérialité, des 
imaginaires littéraires comme ceux de l'Amérique - $a$ fortiori au XIX siècle, période où les romanciers populaires affectent encore de reproduire, au moins comme idéal, une certaine culture du livre et des belles-lettres - demeurent en tension avec les sources de leur mémoire culturelle, faite de textes, d'images, de doxas et d'intuitions non réductibles à leur support médiatique d'accueil, mais y prenant forme ou s'y ajustant de façon complexe et originale ${ }^{2}$.

Indépendamment de ces réserves, ces deux perspectives participent indéniablement à l'objectivation théorique et encyclopédique du roman d'aventures sauvagiste, et c'est en tenant compte de leurs acquis que nous aimerions y revenir ${ }^{3}$. Sans prétendre à l'exhaustivité, nos remarques mettront en avant le caractère délibéré de certaines figures à l'appui desquelles ce roman opère la conversion de tout un pan des représentations françaises de l'Amérique aux codes du romanesque populaire. À travers l'héroïsme du coureur des bois et la qualité d'espace - de chronotope - dans lequel celui-ci se reflète et se meut aventureusement, le roman sauvagiste se donne les moyens de perpétuer des valeurs (et des contradictions) issues du temps long de l'histoire culturelle, tout en les incorporant dans la modernité du récit de masse. Assumer ce point de vue de la rupture des imaginaires au sein de continuités médiatiques nous permettra de mettre en évidence un sous-genre de roman d'aventures qui ne se limiterait pas à une pure variation au sein d'une chaîne médiatique constituée par les catalogues de romans à bas prix. Nous insisterons, en particulier, sur la nécessité de situer la formule aventureuse du roman sauvagiste $a$ minima comme étrangère, sinon comme alternative, à l'héroïsme de la Frontière, et le coureur des bois comme un héros « anti-westernien » par anticipation.

\section{La passion de l'œil vierge : brève archéologie imaginaire du sauvagisme ${ }^{4}$}

6 Des nations européennes, la France des Lumières et préromantique fut sans doute la plus fidèle à l'idéalisation discursive et artistique de l'Indien d'Amérique (Sozzi). Des Dialogues (1704) de Lahontan jusqu'aux proses indiennes de Chateaubriand (Atala, 1801; Les Natchez, 1826), en passant par les critiques, philosophes et polémistes (Le Huron ou l'Ingénu de Voltaire, 1767), une solide tradition s'attache à halluciner l'espace verdoyant où vit l'Amérindien comme une parenthèse virginale sans autre exemple dans le monde. Sans qu'ils soient spécialistes des Amériques, nombre d'auteurs du XVIII ${ }^{\mathrm{e}}$ siècle partagent cette doxa selon laquelle le Nouveau Monde abrite une exceptionnelle zone franche, dernier exemple d'une sauvagerie primordiale. Ses Indiens prospèrent et se régénèrent dans cet éden présumé inviolé et qui abolit, sur son étendue, toutes les frontières anthropologiques et territoriales dans lesquelles l'Europe, alors plus que jamais, est corsetée. Alors que la guerre de Sept Ans sature le Vieux Continent de frontières et d'historicité, la tentation est d'autant plus grande de projeter outreAtlantique l'utopie d'un espace autogéré d'autochtones heureux.

7 En parlant d'une "nostalgie de l'œil vierge », Marc Fumaroli a particulièrement bien résumé les déterminations profondes de cette passion indienne (1999 15). La virginité largement spéculative projetée d'Europe sur l'Amérique indienne impliquait au moins autant le regard que l'objet contemplé. Il s'agissait au fond d'appeler sauvagerie un point de vue extérieur voulant s'abolir lui-même, un regard sur l'Ailleurs voulant se déprendre des médiations qui le font exister. Peindre l'autonomie de la vie sauvage en 
trompant son propre regard d'Européen constitue peut-être le geste fondateur de l'imaginaire sauvagiste, quels que soient ensuite les registres, genres et supports qui véhiculent son expression. Quelques décennies après ses débuts discursifs et rousseauistes parmi les philosophes, ce patron sauvage trouve ses couleurs esthétiques chez les lyriques - via les fresques américaines de Chateaubriand, qui lancèrent toute une vogue transartistique du lamento indien (Bellenger 121) - et ses principes narratifs dans le roman du XIX siècle.

Avant le milieu du XIX ${ }^{e}$ siècle, le sauvagisme était demeuré savant en France, et s'était surtout traduit dans les registres poétique et iconographique. Lorsqu'il investissait le roman, ce n'était encore que d'une façon transposée, en réacclimatant par exemple les chasseurs des romans de l'Américain James Fenimore Cooper (1789-1851) parmi les personnages des Chouans de Balzac ou des Mystères de Paris d'Eugène Sue. Il faut attendre la ruée vers l'or (1848-1849), ses migrations et ses échos dans la presse pour qu'une veine durable de feuilleton d'aventures vienne rattacher autrement la France à ces spéculations du XVIII ${ }^{e}$ siècle. Parallèlement à son entrée dans le roman, la passion indienne déborde alors sur un personnage apparenté au Peau-rouge, mais jusque-là oublié : le coureur des bois ou trappeur. Autrefois honni, cet hybride, à la croisée des mœurs coloniales et amérindiennes au Canada, apparaît soudain comme un médiateur plus idéal que l'Indien lui-même, satisfaisant les passions culturelles liées à la virginité sauvage tout en entretenant chez le lecteur un minimum de familiarité française. Près de cent ans après la perte du Canada par la France, son rejeton sauvage devient la clé de voûte du roman sauvagiste.

Le roman d'aventures sauvagiste sera examiné ici selon deux aspects principaux : l'on considérera d'abord le coureur des bois comme un personnage d'aventures défini par une certaine tradition culturelle; l'on s'intéressera ensuite à la formule aventureuse originale dans laquelle s'illustre ce personnage, et notamment les codes imaginairement très significatifs qui médiatisent ses déplacements et son triomphe dans l'espace. Par ailleurs, et pour donner mieux à voir les implications de l'inscription d'un imaginaire dans un support, l'on comparera régulièrement les figures des romans d'Aimard avec celles d'un autre imaginaire de l'Ouest, lui aussi aventureux, alors en cours de façonnement aux États-Unis: le mythe national de la Frontière, fourrier du pionnier et de la grammaire narrative et visuelle du western ${ }^{5}$.

\section{Le coureur des bois, succès tardif d'un héroïsme français}

On ne peut nier la part de substrat historique qui entre dans les clivages ou contrastes entre imaginaire sauvagiste et mythe de la Frontière. Loin des colonies de peuplement structurées de Nouvelle-Angleterre, l'univers dont est issu le coureur des bois francocanadien - et dont s'inspire probablement aussi le Bas-de-Cuir de Cooper, en dépit des dénégations patriotiques de son auteur - est celui d'une colonisation française qui resta longtemps indifférente à la conquête terrestre et à l'exploitation intensive de ses territoires américains. Aux XVII ${ }^{e}$ et $\mathrm{XVIII}^{\mathrm{e}}$ siècles, les forts et comptoirs disséminés en Nouvelle-France étaient rares, et les annuelles foires de commerce, insuffisantes pour fournir les pelleteries attendues d'un si grand empire. Les traiteurs de fourrures s'avéraient contraints de voyager en solitaires pour s'adjuger le concours des chasses amérindiennes et en négocier les prises : on les appelait alors coureurs de bois ${ }^{6}$. Lorsque 
l'un d'entre eux se faisait lui-même chasseur, seul ou en équipe, on parlait alors de trappeur ou de voyageur.

Hors des villes de la Nouvelle-France, cette absence d'exploitation intensive faisait que les rapports aux nations algonquiennes avoisinantes excluaient toute culture du clivage ou de l'affrontement. Le colon français devait traiter, à tout point de vue, avec l'Indien, voire s'allier et «convivre » avec lui pour emporter ses faveurs. Économie et société se bâtissaient autour de cet enjeu, conférant à la colonie une morphologie particulière ainsi qu'une certaine originalité de mœurs et de pratiques. Avec la notion de Middle Ground, Richard White va jusqu'à concevoir le fonctionnement de l'Amérique française comme une négociation ethnoculturelle continue au sein de laquelle, finalement, l'apport français et l'apport indien se compénètrent et se confondent. Dans ce contexte de consensus devant pallier la désinvolture française en matière d'occupation et d'appropriation des terres, la Nouvelle-France survivait grâce à des médiateurs qui, comme le coureur des bois, étaient capables de se déployer au plus profond des forêts du continent pour perpétuer le Middle Ground et approvisionner la colonie en pelleteries. Trait culturel déjà marquant : c'est parce que sa souveraineté en Amérique restait largement nominale que la France, jusque dans son style colonial, avait dî préférer l'espace au territoire. La mobilité du coureur des bois était à la fois permise et requise par le flou des frontières, et non par «la » Frontière perçue comme clivage mythique.

12 Le coureur franco-canadien n'a été construit qu'assez tardivement (et, on va le voir, sous l'effet d'influences extérieures) comme héros de roman d'aventures. Cet avènement différé a pour première conséquence de désindexer le personnage de sa stricte historicité d'origine. L'imaginaire français n'incorpore véritablement son ancien hybride colonial qu'au milieu du XIx $x^{e}$ siècle, à l'heure où l'espace américain, théâtre d'enjeux politiques qui laissent la France hors-jeu, ne présente plus depuis longtemps les conditions qui avaient vu prospérer le coureur des bois aux XVII et XVIII ${ }^{\mathrm{e}}$ siècles. Son retour apparaît en cela comme un surgissement sans tradition antérieure dans la littérature française. Et pour cause : l'Ancien Régime s'était toujours méfié de cet agent qu'il peinait à contrôler. La promiscuité qu'il entretenait avec le monde amérindien jusqu'à la perpétuation de dynasties de Métis (les Bois-Brûlés) - horrifiait les autorités coloniales. En cela, le fait que le terme "coureur des bois" ait connu sa première occurrence dans un texte juridique répressif (Havard 10-11) est lourd de signification. Malgré son utilité économique, ce traiteur demeurait un homme "sans aveu». Incontrôlable par l'État, il semble l'être aussi pour la littérature. L'hermaphrodisme que Balzac salue en lui constitue précisément ce qui, au siècle précédent, faisait de lui un repoussoir. Trop «blanc» pour bénéficier d'une idéalisation naturelle comme le Bon Sauvage, trop compromis dans la vie indienne pour mériter une épopée nationale, le coureur des bois traversera les Lumières comme une matière stérile pour le mythe autant que pour le roman. Dans le meilleur des cas, les écrivains se contentent d'un silence pudique, au pire ils propagent à son endroit une exécrable réputation, perceptible jusqu'à Chateaubriand.

13 Il faut donc attendre plusieurs décennies pour que le coureur des bois se retrouve idéalisé, comme l'Indien, dans les codes d'un imaginaire sauvagiste et français à la fois. La France, cependant, n'aurait sans doute pas redécouvert le potentiel culturel de ce personnage sans l'intervention de deux médiateurs étrangers : Lord Byron et (surtout) James Fenimore Cooper. Dans un passage du chant VIII de Don Juan, le premier se saisit 
du célèbre chasseur américain Daniel Boone en l'expurgeant de tout caractère historique. Ce rude pionnier qui avait ouvert le Kentucky aux jeunes États-Unis y devient un impalpable « enfant de la nature » («Child of Nature ») s'ébrouant dans un labyrinthe sylvestre («deepest maze») qui l'affranchit et l'enclave à la fois. Pour la première fois, un chasseur blanc - même s'il n'est pas français - se voit idéalisé comme un Bon Sauvage. L'Américain James Fenimore Cooper devait en retenir la leçon. Natty Bumppo, le héros des Leatherstocking Tales, est la traduction romanesque de cette figure de colon paradoxal immergé dans une nature dont il finit par devenir l'appendice. Si Natty Bumppo non plus n'est pas franco-canadien et qu'il tient à se prévaloir d'un "sang pur ", il est bien ce chasseur qui vit dans l'Ouest sauvage avec des Amérindiens qu'il traite comme ses semblables au point de devenir hostile à ses propres compatriotes, les pionniers américains : reculant à mesure que ceux-ci progressent, Natty vagabonde de roman en roman en quête d'un dernier espace aéré. Ce qu'invente le roman de Cooper, dès lors, ce n'est pas le progressisme pionnier, mais un modèle romanesque prenant comme par avance le contrepied du western. L'ordre même de parution des romans du cycle, de The Pioneers à The Deerslayer, prend une direction antichronologique en résonnance avec la quête primitive du héros : tout ramène Natty Bumppo - cheminant à contre-conquête et retrouvant, à contre-histoire, une manière d'innocence de l'aventure - à l'ingénuité et à l'enfance naturelle qu'évoquait déjà le poème de Byron. Lauric Guillaud voit dans ce mouvement l'expression délibérée d'une "plongée régressive " passant par "l'évocation d'un passé archaïque de plus en plus imaginaire et la peinture d'une nature ouvertement symbolique » (Guillaud 52).

Plus que le mythographe des États-Unis, Fenimore Cooper aura donc été un adaptateur de traditions européennes aux thèmes de l'Amérique. À l'image de ses innombrables épigraphes de chapitres tirées de Shakespeare, ses sources, comme le rappelle Richard Slotkin, sont essentiellement européennes : " [they] are essentially European, as are the attitudes it embodies. The Romantic heroes and heroines, and their foils accomplices, are almost always stereotypes, two-dimensional stack figures from Scott or the novel of manners » (Slotkin, 1973 486). Cette perpétuation d'un rapport centripète aux modèles et prescriptions de l'ancienne métropole n'est pas pour rien dans la si favorable réception dont Cooper bénéficia en Europe. Le cas de la France, pays où l'écrivain résida plusieurs années et où il trouva ses plus ardents défenseurs, apparaît emblématique de cette dépendance culturelle persistante. Dans ses articles consacrés à Bas-de-Cuir, Balzac, on l'a vu, salue le «magnifique hermaphrodite moral » (268). D'autres éloges, comme celui de Barbey d'Aurevilly, apparaissent tout aussi significatifs. En louant, chez Cooper, celui qui a compris que "l'Amérique ne vaut que quand elle est un désert, une forêt, une chose antédiluvienne et sauvage, [...] une solitude du cinquième jour de la Création » (17), le Connétable relève un trait récurrent de l'image française de Cooper : celle d'un romancier qui, par le choix de l'intégrité sauvagiste, avait su rester lucide, et même entrer en dissidence avec le matérialisme de ses compatriotes. En inventant Basde-Cuir, Cooper montrait donc patte blanche imaginaire: son cycle romanesque semblait tout entier arriver en renfort de la passion de l'œil vierge, avec d'autant plus d'autorité que l'auteur en était américain.

Autre enthousiaste de Cooper à qui elle consacra plusieurs articles, George Sand fut, en même temps, la préfacière du Coureur des bois de Gabriel Ferry. C'est ce romanfeuilleton, paru dans L'Ordre en 1850 et recueilli en volume en 1853, qui, par le truchement du modèle de Bas-de-Cuir, marque l'entrée différée en littérature du chasseur canadien. Celui-ci, dans le même temps, voit ses aventures déplacées du 
bassin de l'Ohio vers le Sud-Ouest des États-Unis. En effet, la région allant de la Californie aux états frontaliers du Mexique était entre-temps devenue le catalyseur des projections américaines des Français (Venayre). D’abord adossé à la ruée vers l'or dans le désert de Sonora, le roman finit par s'en émanciper pour ne retenir d'essentiel que la peinture de son héros, Bois-Rosé. Le chasseur canadien d'origine bretonne, réimplanté dans les borderlands mexicains, n'en conserve pas moins l'habit traditionnel des coureurs des bois de la Nouvelle-France du XviII ${ }^{\mathrm{e}}$ siècle :

[Bois-Rosé] portait un vêtement qui tenait à la fois de celui de l'Indien et du Blanc. Il était coiffé d'un bonnet en forme de cône tronqué, fait en peau de renard. Une chemise de coton à raies bleues couvrait ses épaules ; à côté de lui, par terre, était déposée une espèce de surtout fait d'une couverture de laine. [...]

Une corne de buffle soigneusement grattée était passée en sautoir sur ses épaules et contenait sa poudre, tandis qu'un sac en cuir, qui faisait pendant à la poudrière, renfermait une abondante provision de balles de plomb. Enfin, une carabine ou rifle à long canon, déposée près de lui, un couteau de chasse passé dans un baudrier ou plutôt dans une ceinture de laine de diverses nuances, composaient son équipement de campagne (Ferry 192-193).

Par son habit anachronique et le style de vie qu'il partage avec le noble Indien, le coureur des bois parait forgé pour une diégèse qui ne serait que sylvestre et sauvage, au lieu de quoi le roman l'associe constamment aux aventures des chercheurs d'or (les gambusinos), des brigands (salteadores) et des bouviers (vaqueros), sans oublier ces pronunciamientos militaires qui sont monnaie courante dans l'instable république mexicaine. Compromis dans ces intrigues, Bois-Rosé leur demeure toutefois étanche, comme issu d'un tout autre moule imaginaire. Ce clivage actanciel, s'il frôle parfois l'erreur de composition, s'avère lourd de sens au plan imaginaire : Le Coureur des bois campe un chasseur franco-canadien conditionné pour l'aventure sauvagiste, mais l'aventure elle-même continue de s'insérer dans un espace territorialisé - un espace que Ferry, voyageur chevronné, chroniqueur pour la Revue des Deux Mondes et romancier bourgeois, répugnait à déréaliser complètement (Letourneux).

\section{Le franc trappeur de Gustave Aimard}

Si Gabriel Ferry, dans le sillage de la très favorable réception de Cooper en France, avait redécouvert le coureur des bois en tant que sauvage blanc et francisé, c'est bien Gustave Aimard (1818-1883) qui, avec ses dizaines de romans, fera du personnage un standard du roman populaire de l'époque. L'abondante production d'Aimard rendra véritablement aventureux le parcours régressif de Bas-de-Cuir tout en radicalisant le sauvagisme encore «négocié » de Ferry. Par ailleurs, en faisant entrer de plain-pied le sauvagisme dans la production feuilletonnesque, Aimard stabilisera les codes sauvagistes dans un sous-genre visible et adapté au ressassement sériel (Nathan 1990). Ce faisant, malgré les répétitions et les coquilles malheureuses, Aimard conférera au coureur des bois un patron héroïque solide, sous la forme duquel le personnage perdurera dans les imaginaires français jusqu'à l'entre-deux-guerres. Les chevaliers naturels de ses romans, beaux rejetons du Sauvage, s'appellent Cœur-Loyal, BalleFranche, Sans-Piste ou Belhumeur. Qu'ils soient qualifiés (cela varie souvent) de trappeurs, de chasseurs, de coureurs des bois ou de batteurs d'estrades, tous présentent et répètent invariablement un même type actanciel. On peut fixer ce type dans l'expression de franc trappeur, dont l'épithète nous semble valide à deux titres : ce héros est franc au sens étymologique, puisqu'il évolue dans un espace exempté, une zone 
franchisée, sainement libre; mais il est encore franc au sens où sa mise en intrigue entend réaffirmer une francité américaine, témoignage perdu d'une nation qui avait su être souveraine dans le Nouveau Monde sans faire offense à la virginité du continent. Chez Aimard, cette volonté de diffuser une certaine idée de l'Ouest à travers la France ne souffre d'aucune ambiguïté. Forgé comme le médiateur d'une Amérique antérieure aux pionniers - et même " antéprédicative ", émancipée de toute frontière humaine -, le franc trappeur déploiera un héroïsme sans rapport avec l'idiosyncrasie coloniale anglo-américaine.

18 Au physique, les trappeurs d'Aimard portent à incandescence les codes de la tradition antérieure. Le costume porté par Cœur-Loyal se ramène à un agrégat d'oripeaux tout juste assemblés. Son équipement, en revanche, emprunte presque tout aux attributs du noble médiéval, ici transposés dans un matériel cynégétique à haute valeur symbolique. Le chapeau en peau de loutre ou de castor, les mocassins, la gibecière, la sacoche de poudre et l'archaïque rifle constituent ainsi un attirail analogue aux armes et talismans des chevaliers. D'autre part, pour mal fagoté qu'il soit, le chasseur n'en observe pas moins la conduite d'un aristocrate sylvestre : fidèle à son nom, Cœur-Loyal sacrifie tout à son devoir, y compris les avances timides qu'il fait, en galant courtois, à la jeune Doña Luz. Bon-Affût est pour sa part un modèle de «physionomie ouverte, franche et loyale qui appelait la sympathie et plaisait de prime abord " (Aimard, 2001 484). Chez Aimard, le franc trappeur fait culminer la noblesse supposée de l'ensauvagement: l'expression «âme (ou nature) d'élite » est récurrente sous sa plume pour qualifier son personnage. À travers ces emprunts littéraux au chronotope du chevalier errant (Bakhtin 298-304), le personnage se construit avant tout comme projection, sur une Amérique déjà idéalisée, de structures parmi les plus anciennes du roman européen. L'archaïsme propre à l'état de nature se réalise ainsi dans un autre archaïsme : celui des modèles convoqués pour lui dégager des voies romanesques.

Le franc trappeur demeure cependant un Indien blanc avant tout, et ce sont ses rapports mimétiques avec le noble Sauvage qui le qualifient pour l'aventure. On peut citer l'usage, ici encore « industrialisé » par Aimard, de noms totémiques : Bois-Rosé, Balle-Franche, Sans-Piste ou Poignet-d'Acier sont autant de sobriquets qui, en les "baptisant », convertissent les coureurs des bois à l'ordre sauvagiste de la Prairie. Frères des Peaux-Rouges, ils n'affrontent jamais ces derniers que par escarmouche, car ils leur sont liés. Si ce trait présidait déjà à l'amitié entre Uncas et Natty Bumppo dans Le Dernier des Mohicans, ou à celle unissant Bois-Rosé et Rayon-Brûlant dans Le Coureur des bois, Gustave Aimard va souvent plus loin dans l'expression d'une proximité entre trappeurs et Indiens. Chez lui, les scènes d'alliances rituelles et d'échanges des sangs sont légion. Dans Le Chercheur de pistes, les Comanches adoptent solennellement Valentin, coureur des bois d'origine française, et dans Les Pirates des prairies, suite du précédent, c'est aux Apaches qu'il s'unit. Aimard prétendait lui-même avoir épousé une Comanche dans le cours de ses voyages, et ne boude pas non plus les mariages mixtes : en témoigne l'union entre Numank-Charakè et Rosée-du-Soir, jeune sauvagesse de sang-mêlé, dans Les Outlaws du Missouri (1868). Enfin, il est le seul de nos écrivains chez qui la défense des vieilles colonies françaises se traduit dans la réhabilitation du BoisBrûlé, métis de Blanc et d'Indienne, brocardé au XvIII siècle. C'est le cas dans le cycle d'aventures consacré au trappeur Balle-Franche, mais aussi dans plusieurs romans à prétention « historique » (La Belle Rivière, 1874 ; Le Souriquet, 1882) destinés à répondre à la version de la guerre de Sept Ans popularisée par Cooper. Aimard n'hésite pas à y 
exalter le rôle patriotique des Métis franco-canadiens : «Les plus hardis coureurs des bois, les plus audacieux explorateurs de ce temps, tous, quoi qu'on en ait dit, ont été des bois-brûlés. Lorsque parfois on leur demande à quelle nation ils appartiennent, ils répondent avec fierté qu'ils sont Français et ils ont raison! » (Aimard, 1894 339).

\section{Le trappeur et la « lutte des traces » : déroute, pistage et narrativité}

Il est un autre trait de son "comportement narratif», peu mis en évidence dans les travaux existants, qui confère au franc trappeur son relief héroïque particulier et le rend inassimilable (tant au plan historique que générique) aux cow-boys et autres héros de la Conquête de l'Ouest. Dans les descriptions romanesques comme dans l'iconographie ${ }^{7}$, le coureur des bois se donne le plus souvent à voir adossé à une souche ou appuyé sur son rifle, en posture d'attente, d'observation ou de sagesse, c'est-à-dire selon autant d'instantanés qui dénoncent l'homme à pied. S'il est un chevalier, le trappeur l'est sans sa monture, préférant l'approche d'un marcheur à l'affût aux « charges héroïques » et autres « chevauchées fantastiques » qui feront les beaux jours du western. En plus de le rapprocher d'une image particulièrement archaïque de l'Indien, cette posture de dénuement induite par la marche confère un sens esthétique à l'aventure. Opter pour l'héroïsme "piéton » de l'homme à l'affût revient en effet à s'assurer qu'aucun expédient agressif, pas même le cheval, ne vienne corrompre l'harmonie entre l'aventurier sauvagiste et les vastitudes où il évolue. Dans Le Dernier des Mohicans, déjà, Natty Bumppo prophétisait que l'irruption du cheval viendrait marquer un point de bascule dans l'histoire de l'Ouest (Cooper 753). Le Bois-Rosé de Gabriel Ferry se décrit lui-même comme « un triste cavalier » (Ferry 728) et affiche son «mépris pour le cheval » (204). Les trappeurs d'Aimard confirment et systématisent ce topos: dans Le Chercheur de pistes (1858), Valentin et l'Indien Curumilla entendent circuler "selon la coutume indienne ", manière qu'ils jugent "plus sûre et tout aussi prompte que sur un cheval» (Aimard, 1865 169). Bon-Affût, le héros de L'Éclaireur (1859), prône la même attitude et invite ses amis à abandonner leurs montures: «Laissez vos chevaux, ils nous sont inutiles, ce n'est pas un voyage que nous entreprenons, nous commençons une chasse à l'homme; il nous faut être libres de nos mouvements; la piste que nous suivons est difficile» (Aimard, 2001 661). «[D]ans la montagne, dit encore l'un des personnages de La Loi de Lynch (1859), un piéton est partout, un cavalier nulle part» (Aimard, 1863 424). Tout sauf contingente, cette exclusion du cheval des habitudes du héros participe délibérément de l'imaginaire sauvagiste, puisqu'il s'agit de garantir l'inviolabilité de l'Amérique contre toute circulation " par effraction ». En cela, le contraste demeure flagrant avec le fétichisme équestre de la Frontière et ses corollaires (pensons au «Iron Horse » qui désigne le train dans l'Ouest).

21 Le mode de circulation de ce trappeur-piéton se réfléchit dans les qualités mêmes de l'espace qui lui sert de terrain d'aventure. On peut continuer d'évoquer cet espace dans les termes du chronotope dans la mesure où Aimard lui-même, par une sorte d'aveu méta-générique, parle du "système du désert » (Aimard, 2001 240) ${ }^{8}$. Une première strate de la spatialisation aventureuse du sauvagisme concerne l'esprit de la déroute. L'espace connu dans ces romans sous le vocable de prairie, antérieurement à tout paysage particulier, correspond à un périmètre générique (et non-géographique) ayant 
pour vocation de perdre son visiteur. Si c'est une forêt vierge, elle rejoint les cryptages ténébreux du roman gothique : « La perspective n'existe pas, c'est à peine si la vue peut s'étendre à trente ou quarante pas dans toutes les directions" (Aimard, 1863 294); et ses lianes «qui courent d'arbre en arbre » forment « en se croisant et s'enchevêtrant les unes dans les autres une barrière presque infranchissable, comme si la nature jalouse avait voulu dérober aux regards profanes les mystérieux secrets de ces forêts " (Aimard, 2001 540-541). Si c'est un désert, son horizon épuré reste tout de même un " dédale » (Aimard, 1864 135), mais un dédale ouvert au plein air (dédale anti-gothique, en somme), "où les routes n'existent pas » (45). Quel que soit son décor, l'espace sauvagiste présente toujours une touffeur ou une vastitude qui effacent a priori les traces et tracés humains. Sa densité abstraite oblige les héros, trappeurs et Indiens, à circuler en son sein de façon non-linéaire et non-immédiate. "Au désert, si l'on veut aller au nord, il faut marcher vers le sud » (Aimard, 1863 285-286); « dans le pays où nous sommes la ligne droite est toujours la plus longue » (Aimard, 1864 22). Toutes ces notations configurent avec insistance l'Ouest d'Aimard comme un espace de déroute dans un écrin naturel.

Dans L'Éclaireur, il est préconisé au trappeur de « louvoyer », d'avancer par zigzags en imitant « la manœuvre du navire » (Aimard, 2001 663). Ici, les dérives du navire en mer et celles du trappeur dans la prairie se ressemblent dans leur refus des routes, des tracés téléologiques que le western, au contraire, voudra figer dans le marbre du mythe en surdéterminant des figures comme le cheval, la diligence ou la voie ferrée. De ce point de vue, le coureur des bois doit plus au marin romanesque qu'au cow-boy. Dans l'œuvre de leurs auteurs, les romans sauvagistes côtoient d'ailleurs souvent des romans maritimes au XIX ${ }^{e}$ siècle - chez Fenimore Cooper, par exemple, Les Pionniers (1823) précède Le Pilote (1824) - tandis que les trappeurs sont régulièrement présentés comme des matelots s'orientant sur la terre ferme. C'est le cas dans Le Coureur des bois: "Combien, se disait Bois-Rosé en suivant le cours de ses idées, combien l'Océan ressemble au désert ! J'entends d'ici comme la mer qui brise, j'entends d'ici le canon qui retentit au large. Combien de fois le craquement des mâts ne m'a-t-il pas rappelé le frémissement de ces grands arbres?» (Ferry, 2009 475). Ancien marin comme BoisRosé, Olivier, héros récurrent de Gustave Aimard, connaît ce même type de reconversion de la navigation maritime à la «navigation » terrestre.

23 À la faveur de cette propension à la dérive, le franc trappeur a également pu être rapproché d'autres figures romantiques et européennes de l'errance en vogue au milieu du siècle. Dans l'inspiration du Coureur des bois de Gabriel Ferry, Mark Wolff a identifié le hasardeux tâtonnement du flâneur baudelairien (Wolff 143-156). Les écrivains euxmêmes, pour leur part, n'hésitent pas à brandir la figure du juif errant : les trappeurs du Défilé d'enfer (1891) de Louis Boussenard sont «nomades à rendre des points au JuifErrant lui-même » (Boussenard 335); et Arnold de Woelmont, déplorant le destin du chasseur qu'il croise sur sa route, s'exclame: «Va donc, pauvre juif errant!» (Woelmont 126). Au sein d'un sous-genre romanesque comme le mystère urbain, le flâneur et le juif étaient en effet deux incarnations urbaines de l'errance. Le trappeur, qui leur est associé, peut donc se hisser, tout américain qu'il soit, au niveau des grands déroutés du romantisme européen. De quoi confirmer les dettes intergénériques, mais aussi la texture toute littéraire, du roman sauvagiste français.

L'Ouest romanesque sauvagiste, on l'a dit, est un espace vaste et déroutant, mais il ne cesse pas pour autant de solliciter la lecture. Son chaos se veut un chaos provisoire, 
institué à la seule fin de son décryptage par un héros qui sache lire (invitant le lecteur, par cette délégation, à lui aussi se prendre au jeu). En l'absence de route humaine, la prairie déroule un parchemin cosmique, laissant affleurer, à sa surface, maints signes et traces à peine sensibles. Elle se donne comme un damier sémiologique à la fois discontinu et subtilement ordonné : «Tout a une raison d'être dans la prairie, tout est analysé et commenté : une feuille ne tombe pas d'un arbre, un oiseau ne s'envole pas, sans que l'on sache ou l'on devine pourquoi la feuille est tombée, pour quelle raison l'oiseau a pris son vol » (Aimard, 2001 276). Par leur redondance, ces topoï font entrer le lecteur dans le régime d'action abstrait, oblique et indirect qui caractérise l'aventure sauvagiste. À l'héroïsme du défrichage souvent propre au pionnier, les romans de Gustave Aimard préfèrent un héroïsme du déchiffrage. Quand la Frontière magnifie les traceurs de routes et les éventreurs de montagnes, la prairie du franc trappeur récompense l'adresse indiciaire. Cette vertu zigzagante distingue les coureurs des bois comme des aventuriers « analystes » s'orientant par détection dans l'espace naturel.

Façonné dans un creuset intergénérique qui le rattache à des figures préromantiques et romantiques (Indien, flâneur, marin, juif errant, etc.), le franc trappeur s'attache ainsi un ethos de lecteur qui tempère et parfois dépasse son ethos de guerrier. Cet ethos lectoral se manifeste avec insistance au plan métaphorique. Aussi bavards que les Bons Sauvages sur leur propre primitivisme, les coureurs des bois aiment pontifier sur le livre organique. Ils célèbrent la forêt américaine comme un palimpseste qui n'a pas besoin de manuscrit, déployant dans la nature, la seule sagesse, la seule Écriture dignes de ce nom: "Les mocassins ne se ressemblent pas plus que les livres, proclame Natty Bumppo, quoique ceux qui lisent le mieux dans ceux-ci ne soient pas les plus capables de bien distinguer ceux-là » (Cooper, 2003 615-616) ${ }^{9}$. Les chasseurs de Gabriel Ferry sont eux aussi «accoutumés à lire sur [la] surface [de la Prairie] comme dans un livre ouvert" (Ferry 388). Gustave Aimard montre encore plus de zèle dans ce registre métaphorique, par exemple lorsqu'il désigne toute l'Amérique comme le "plus beau livre [...] écrit par Dieu lui-même dans les plaines, sur les montagnes, et jusque dans le moindre brin d'herbe » (Aimard, 1876 27).

Par ailleurs, sur le plan de la conduite narrative de l'aventure, cet ethos s'accompagne d'une forme d'intrigue fidèle au principe des nouvelles déductives d'Edgar Allan Poe, traduites par Baudelaire à la même époque. Carlo Ginzburg a bien mis en évidence, dans ces récits, l'indissociabilité entre l'action de traque et le récit que l'on peut en donner, principe que mobilisent les romans sauvagistes dès Fenimore Cooper. Remontant au « «geste peut-être le plus ancien de l'histoire intellectuelle du genre humain : celui du chasseur accroupi dans la boue qui scrute les traces", le "paradigme indiciaire " (Ginzburg 151) tel que le formalisent les romans d'aventures sauvagistes offrent ainsi leur pendant archaïque aux raisonnements qu'on trouve dans les nouvelles déductives et urbaines de Poe. Dans cet exemple tiré du Dernier des Mohicans, Natty Bumppo re-narrativise le parcours de deux jeunes filles qui viennent d'être capturées par les Indiens pendant que son ami Uncas reconstitue leur piste :

Uncas fut assez hardi, répondit le chasseur en jetant un regard d'intérêt et de curiosité sur les chevaux des deux sœurs, pour nous assurer que les montures de ces dames plaçaient à terre en même temps les deux pieds du même côté, ce qui est contraire à l'allure de tous les animaux à quatre pieds ou à quatre pattes que j'ai connus, à l'exception de l'ours. Et cependant voilà deux chevaux qui marchent de cette manière, comme mes propres yeux viennent de le voir, et comme le prouvaient les traces que nous avons suivies pendant vingt longs milles. [...] Mais n'importe quelle soit l'allure de ces animaux, Uncas l'avait remarquée, et leurs 
traces nous conduisirent à un buisson près duquel était l'empreinte du pied d'un cheval, et dont la plus haute branche, une branche de sumac, était cassée par le haut à une élévation qu'on ne pouvait atteindre qu'à cheval, tandis que celles de dessous étaient brisées et froissées comme à plaisir par un homme à pied. J'en conclus qu'un de ces rusés, ayant vu une de ces jeunes dames casser la haute branche, avait fait tout ce dégât pour faire croire que quelque animal sauvage s'était vautré dans ce buisson (Cooper 550-551) ${ }^{10}$.

Paradoxalement, il semble que ce soient les romans les plus industriellement fabriqués, et notamment ceux d'Aimard, qui formulent de la manière la plus insistante, mais aussi la plus abstraite, cette primauté de la trace dans l'aventure. Ces récits dénudent et dupliquent la ressource indiciaire en une quantité de ricochets, pistes déroutantes, scènes d'affût et retours de traque. Par esprit d'analogie, on pourrait définir le principe de l'action dans un tel chronotope comme une inexorable lutte des traces. La ressource indiciaire typifie la plupart des épreuves; elle fait connaître à l'action autant de relances et de péripéties qu'il en faut au roman-feuilleton pour se prolonger. La diégèse en vient à ressembler à un plateau sémiologique presque conceptuel, chacun des pisteurs s'attachant, à chaque nouvelle reprise de l'aventure, à préserver un «coup indiciaire "d'avance sur ses adversaires. Toutes les marches allument des contremarches, et le moindre enlèvement ne sert bien souvent que de prétexte à la réactivation de ce jeu indiciaire. Il n'est pas rare qu'un même roman, comme Les Trappeurs de l'Arkansas, se structure tout entier autour de plusieurs enlèvements successifs.

On peut affiner cette analyse en parcourant quelques-unes des nombreuses variantes de cette typification indiciaire de l'aventure. Le premier cas, le plus classique, est celui de la piste à retracer elle-même. Son déchiffrage constitue un exercice de sensation et d'intellection requérant ascèse et temporisation. Dans La Loi de Lynch, Aimard explique : "Cette marche pénible était nécessairement lente à cause des précautions innombrables que les fugitifs étaient obligés de prendre pour ne pas être aperçus ou dépistés » (Aimard, 1863 102). Le coureur Valentin Guillois, dans un autre roman, se fait le pédagogue de sa propre pratique : « lorsqu'on suit une piste, il faut faire attention où l'on pose le pied et ne pas regarder ainsi de côté et d'autre " (Aimard, 1864 35-36). Le récit insiste sans arrêt sur la stimulation intellectuelle qu'éprouvent les chercheurs de traces à l'idée d'affronter une piste hermétique :

Valentin et ses deux compagnons avaient beau interroger le désert, le désert restait muet et impénétrable comme un livre fermé.

Depuis cinq heures déjà ils marchaient sans que rien fût venu donner un corps à leurs soupçons et leur prouver qu'ils étaient sur la bonne voie.

Cependant, avec cette patience qui caractérise les hommes habitués à la vie des prairies, et dont aucun mot ne peut exprimer la ténacité, les trois hommes marchaient toujours, s'avançant lentement, pas à pas, le corps courbé, les yeux fixés sur le sol, ne se rebutant pas des difficultés insurmontables qu'ils avaient à vaincre, mais au contraire excités par ces difficultés mêmes qui leur prouvaient qu'ils avaient affaire à un adversaire digne d'eux (Aimard, 1864 340-341).

Dans d'autres cas, une piste trop simple à suivre peut dissimuler un piège. Valentin Guillois évente facilement cette ruse indiciaire dans Les Pirates des prairies - « le CèdreRouge a voulu être trop fin : nous montrer une trace, c'est ce qui l'a perdu » (Aimard, 1864 342) - et Cœur-Loyal et Belhumeur, dans Les Trappeurs de l'Arkansas, se méfient du sillon malin, «parfaitement visible » et « en ligne droite » (Aimard, 2001 41), que laisse derrière lui le Comanche Tête-d'Aigle, ce qui leur permet d'anticiper la manœuvre. 
30 S'ils sont des lecteurs aguerris, les héros sauvagistes doivent aussi savoir se rendre illisibles. Cette seconde variante de la lutte des traces impose aux coureurs des bois de savoir dissimuler les signes de leur passage. Dans L'Éclaireur, la furtivité est aussi l'apanage de Bon-Affût : «allez donc trouver à point nommé dans le désert la piste d'un homme comme Bon-Affût? Qui sait où il se trouve en ce moment? Il peut n'être qu'à une portée de fusil de nous comme en être éloigné de cinq cents milles" (Aimard, 2001 494). Cette "indéchiffrabilité» devient discipline comportementale chez le personnage de Nathan-Othan, « une de ces natures supérieures, sur le visage desquelles il est impossible de rien lire et qui dans toutes circonstances sont toujours maîtresses de leurs impressions » (Aimard, 2001 285).

31 La lutte des traces peut enfin changer de terrain lorsqu'elle emprunte les multiples voies d'eau qui quadrillent le continent américain, façon pour le coureur des bois de se (re)faire marin tout en invoquant, dans les eaux douces de l'Ohio, du Saint-Laurent ou du Mississippi, l'ancienne tradition franco-indienne du canot à portage. En plus d'orner l'intrigue de cette mémoire historique, l'élément aquatique, par la fluidité qu'il impose au jeu des traces, confère à la trame indiciaire une expression particulièrement mélancolique. Dans Le Souriquet, l'un des romans de Gustave Aimard se déroulant pendant la guerre de Sept Ans, le héros préconise un voyage en canot pour tromper les Anglais : «l'eau ne laisse pas de pistes, au lieu que dans les bois, quelques précautions que l'on prenne, il est impossible de ne pas laisser de traces» (Aimard, [1883] 359). Cette répétition à la lettre d'une expression célèbre de Fenimore Cooper - «Water leaves no trail » (Le Seven 2016) - dit beaucoup des ambitions d'Aimard vis-à-vis de son devancier : opposer à ce dernier une "contre-vision " historique favorable au génie français, mais en restant fidèle au même paradigme imaginaire.

Un usage aussi délibéré de la trace, thématisé, esthétisé au point qu'il en vienne à définir intégralement l'héroïsme d'un personnage, ne saurait en effet se réduire à la simple translation d'une vision historique, ni être banalisé comme un mécanisme de roman d'aventures parmi d'autres - et cela en dépit, on l'a vu, d'importants emprunts intergénériques. On dira plutôt que le franc trappeur, surhomme de l'innocence, colon indigène, suzerain sans fief, réactive dans l'aventure populaire une série de paradoxes qui sont des corollaires de la passion de l'œil vierge. Il en va de même de son rapport déréalisé à l'Amérique, perçue comme terrain de jeu et de traque. À cet égard, on trouve dans Ève (1913) de Charles Péguy (1873-1914) un vers qui pourrait paraître hermétique s'il ne cristallisait pas aussi bien une sensation de lecture d'enfance : «Et c'est dans les réseaux du plus ancien trappeur" (Péguy 1199). Le coureur des bois romancé du $\mathrm{XIX}^{\mathrm{e}}$ siècle est bel et bien cet aventurier réticulaire, sans noyau ni trajet prédéterminé, dont les prouesses se réalisent hors des logiques de frontalité et d'effraction propres à la Conquête de l'Ouest. À travers lui, Aimard déploie une aventure autogénérée : revenant continuellement sur ses pas, cette aventure paraît aimantée par un centre lumineux perdu qui se serait disséminé dans l'espace. C'est parce qu'elle transpose ce regard dans le contexte du roman populaire qu'une production comme celle d'Aimard parvient, le plus souvent, à maintenir sa vision de l'Amérique à égale distance du regard exotisant de l'exploration coloniale - pensons aux romans d'aventures africaines - et du regard conquérant des pionniers américains. 


\section{Conclusion}

Cette autre Amérique aventureuse que Gustave Aimard popularisa pendant un quart de siècle dans ses romans lui survécut. À la faveur des rééditions, ses romans continuèrent de circuler jusqu'à l'après-guerre. D'Aragon à Michel Le Bris, en passant par Genevoix et Péguy, les coureurs des bois sauvagistes ont aussi régulièrement refait surface dans les souvenirs littéraires d'auteurs importants, parfois en résonnance avec le présent des relations transatlantiques. Ainsi Maurice Barrès, au moment d'évoquer le débarquement canadien de 1915, mobilise en bloc l'imaginaire sauvagiste, de son noyau littéraire à ses marges populaires. Les alliés venus à la rescousse de la France font l'objet d'une vision hallucinée. Les tranchées se muent en sentiers de guerre et les troupes rangées deviennent des essaims de sauvages pratiquant la lutte des traces :

... on trouve dans l'armée canadienne une survivance des vieux romans de la prairie. On m'a montré, sous l'uniforme kaki, des trappeurs qui emploient contre l'ours allemand les mille bons tours de leur métier. Ils chassent le Boche avec l'astuce des anciens chasseurs de chevelures ou du moins des chasseurs de fourrures, adoucies par l'humanité anglaise. "Nos Canadiens, me dit un officier du camp, ont pris immédiatement sur l'ennemi un ascendant moral et matériel sans conteste, au point de vue des patrouilles ». Et il multiplie les détails probants.

Tandis qu'on suit au sillage les Allemands qui rampent dans les champs de blé, les Canadiens savent s'y glisser sans que bouge un seul épi. Ils restent des heures entières à l'affût, couchés sur le dos et regardant derrière eux à l'aide d'un petit miroir. Quand le Boche, rassuré par ce long silence du champ immobile, se hasarde, il est pris, étranglé ou ficelé en deux secondes. L'autre jour, après une série de ces affûts heureux, les «trappeurs " firent parvenir à la tranchée allemande ce simple billet: «Inutile de renvoyer une autre patrouille; ce sont les Canadiens que vous avez en face de vous " (Barrès 291-293).

Il n'est pas jusqu'aux fameux Peaux-Rouges que l'auteur des Déracinés n'intègre à ce tableau militaire. Devant la dépouille d'un engagé amérindien, l'écrivain salue un " authentique représentant de la race célébrée par Chateaubriand, Fenimore Cooper et Gustave Aimard ». C'est, on le voit, l'ensemble de la mémoire sauvagiste, tous types de production confondus, qui se trouve ici rétablie dans sa cohérence et sa transversalité imaginaires. « Une nouvelle fois », renchérit Barrès, « le dernier des Mohicans venait de mourir » (294).

\section{BIBLIOGRAPHIE}

\section{OUVRAGES CITES}

AIMARD, Gustave. La Loi de Lynch. 1859. Paris : Amyot, 1863.

---. Les Pirates des prairies. 1858. Paris : Amyot, 1864.

---. Le Chercheur de pistes. 1858. Paris : Amyot, 1865.

---. Les Outlaws du Missouri. 1868. Paris : Amyot, 1876. 
---. Le Souriquet. 1883. Paris : Fayard frères, s.d.

---. La Belle Rivière. 1874. Paris : Geoffroy, 1894.

---. Les Trappeurs de l'Arkansas et autres romans de l'Ouest [Les Trappeurs de l'Arkansas, 1858 ;

L'Éclaireur, 1859 ; Balle-Franche, 1861 ; Les Bandits de l'Arizona, 1881]. Introduction de Matthieu Letourneux. Paris : Robert Laffont, « Bouquins », 2001.

BAILLEUL, Louis. Le Trappeur du Far-West. 1888. Paris : Lefèvre, 1909.

BAKHTIN, Mikhail. Esthétique et théorie du roman. Paris : Gallimard, « Tel », 2008.

BALZAC, Honoré de. Articles et chroniques. Choix et édition de Marie-Ève Thérenty. Paris :

Flammarion, «GF », 2014.

BARBEY D'AUREVILLY, Jules. « Un Cooper d'Ambigu-Comique ». Voyageurs et romanciers. Paris : Lamerre, 1908, p. 13-18.

BARRÈS, Maurice. L'Âme française et la guerre. vol. 6. Paris : Émile-Paul frères, 1917.

BELLENGER, Sylvain. « Girodet et la littérature, Chateaubriand et la peinture ». Chateaubriand et les arts. Dir. Marc Fumaroli. Paris : de Fallois, 1999, p. 111-135.

BILLINGTON, Ray A. Land of Savagery, Land of Promise. The European Image of the American Frontier in the Nineteenth Century. New York : W. W. Norton \& Company, 1981.

BLETON, Paul. Western, France. La place de l'Ouest dans l'imaginaire français. Amiens : Encrage/Les Belles Lettres, 2002.

BOUSSENARD, Louis. Le Défilé d'enfer. Paris : Flammarion, 1891.

BYRON, Arthur Gordon. The Complete Poetical Works. vol. 5. Ed. Jerome J. McGann. Oxford : Oxford University Press, 1992.

COOPER, James Fenimore. Le Dernier des Mohicans et autres romans du cycle de Bas-de-Cuir. Paris : Omnibus, 2003.

---. The Last of the Mohicans : A Narrative of 1757. 1826. gutenberg.org/files/940/940.txt. Page consultée le 29 avril 2019.

CRACROFT, Richard H. "World Westerns. The European Writer and the American West ». A Literary History of the American West. Dir. Thomas J. Lyon. Fort Worth : Texas Christian University Press, 1987, p. 159-175.

DI GREGORIO, Luca. «Buffalo Bill, une attraction transmédiale ? Brève phénoménologie populaire du circus man de l'Ouest ». Phantasia. vol. 1, 2015. popups.ulg.ac.be/0774-7136/index.php ?id =366. Page consultée le 13 août 2017.

---. « Gustave Aimard ou l'imagerie indienne dans le roman d'aventures ». Le Scalp et le Calumet. Imaginer et représenter l'Indien en Occident $d u \mathrm{XVI}^{\mathrm{e}}$ siècle à nos jours. Dir. Annick Notter. Paris : Somogy, 2017, p. 66-69.

---. « L'imaginaire enfantin de l'Ouest : du petit trappeur au cow-boy ». Le Magasin des petits explorateurs. Dir. Roger Bouley et Julien Bondaz. Arles : Actes Sud, 2018, p. 279-283.

---. Wilderness et Western. L'Ouest fictionnel chez Gustave Aimard et Emilio Salgari. Liège : Presses Universitaires de Liège, 2014.

FERRY, Gabriel. Le Coureur des bois. 1853. Paris : Phébus, 2009.

FOURIER, Charles. Euvres complètes. Tome III. Paris : Société pour la propagation et pour la réalisation de la théorie de Fourier, 1841. 
FUMAROLI, Marc. « Ut Pictura poesis : Chateaubriand et les arts ». Chateaubriand et les arts. Dir. Marc Fumaroli. Paris : de Fallois, 1999, p. 11-42.

---. Paris-New York et retour. Voyage dans les arts et les images. Paris : Flammarion, 2011.

GENEVOIX, Maurice. Laframboise et Bellehumeur. Paris : Flammarion, 1942.

GINZBURG, Carlo. Mythes, emblèmes, traces. Morphologie et histoire. Paris : Flammarion, 1979.

GUILLAUD, Lauric. Frontières barbares. L'espace imaginaire américain de C.B. Brown à Jim Morrison. Paris : E-Dite, 2000.

HAVARD, Gilles. Les Coureurs de bois. Paris : Les Indes Savantes, 2016.

HAVARD, Gilles et AUGERON, Marc, dir. Un Continent en partage. Cinq siècles de rencontres entre Amérindiens et Français. Paris : Les Indes Savantes, 2013.

JACQUIN, Philippe. Les Indiens blancs. Français et Indiens en Amérique du Nord (XVII ${ }^{e}$-XVII ${ }^{e}$ siècle). Paris : Payot, 1987.

LE SEVEN, Emilia, " "Water leaves no trail". Eau, trace et mémoire dans The Last of the Mohicans. » James Fenimore Cooper ou la frontière mélancolique. The Last of the Mohicans et The Leatherstocking Tales. Dir. Agnès Derail et Cécile Roudeau, Paris : Rue d'Ulm, 2016, p. 49-61.

LETOURNEUX, Matthieu. Préface à Gabriel Ferry, Le Coureur des bois. Paris : Phébus, 2009, p. 7-39.

MATHÉ, Roger, «L'image de l'Indien emplumé et du trappeur dans la littérature populaire au XIX siècle ». Popular Traditions and Learned Culture in France. Dir. Marc Bertrand. Saratoga, Calif. : Anma Libri, Stanford French and Italian Studies, 1985, p. 179-209.

NATHAN, Michel. « Le Ressassement, ou que peut le roman populaire ». Splendeurs et misères du roman populaire. Dir. Michel Nathan. Lyon : Presses Universitaires de Lyon, 1990, p. 191-200.

PÉGUY, Charles. CEuvres poétiques et dramatiques. Paris : Gallimard, « Pléiade », 2014.

SLOTKIN, Richard. Regeneration through Violence. The Mythology of the American Frontier, 1600-1860. Middletown : Wesleyan University Press, 1973.

---. Gunfighter Nation : the Myth of the Frontier in Twentieth-Century America. Norman : University of Oklahoma Press, 1998.

SMITH, Henry Nash. Terres vierges. Traduit de l'anglais par Jeanne Collin-Lemercier. Paris :

Seghers, « Vent d'Ouest », 1967.

SOZZI, Lionello. Immagini del selvaggio. Mito e realtà nel primitivismo europeo. Rome : Edizioni di Storia e Letteratura, 2002.

TRIGGER, Bruce G. Les Indiens, la fourrure et les Blancs. Montréal : Boréal, 2011.

TURNER, Frederick Jackson. La Frontière dans l'Histoire des États-Unis. Préface de René Rémond. Traduit de l'anglais par Annie Morin Rambert. Paris : Presses Universitaires de France, 1963.

VENAYRE, Sylvain. « Le moment mexicain dans l'histoire française de l'aventure (1840-1860) ». Revue d'Histoire et Société de l'Amérique latine, vol. 7, 1998, p. 123-137.

VILLERBU, Tangi. La Conquête de l'Ouest. Le récit français de la nation américaine au XIXe siècle. Rennes : Presses Universitaires de Rennes, 2007.

---. Les Missions du Minnesota. Catholicisme et colonisation dans l'Ouest américain, 1830-1860. Rennes :

Presses Universitaires de Rennes, 2014. 
WHITE, Richard. Le Middle Ground : Indiens, empires et républiques dans la région des Grands lacs, 1650-1815. Traduit de l'anglais par Frédéric Cotton. Toulouse : Anacharsis, 2009.

WHITE, Richard et Patricia Nelson LIMERICK. The Frontier in American Culture. Berkeley : University of California Press, 1994.

WOELMONT, Arnold de. Souvenirs du Far-West. Paris : Plon et Cie, 1883.

WOLFF, Mark. « A World of flâneurs and coureurs des bois : Ethnography and the Picturesque in the Works of Gabriel Ferry ». French Literature Series, vol. 23, 1996, p. 143-156.

\section{NOTES}

1. Pensons au mystère urbain, aux romans de cape et d'épée, d'anticipation, fantastiques, d'aventures géographiques, etc.

2. La négation de cette tension au bénéfice des seules continuités médiatiques a pu conduire à certaines propositions assez discutables. Dans son essai encyclopédique Western, France paru en 2002 et couvrant plus de deux siècles de production-réception-traduction de récits de l'Ouest en France, Paul Bleton adopte d'emblée une conception médiatique et englobante du western. Serait «western» tout ce qui aurait été un jour estampillé comme tel, ce qui le conduit à confier la définition de son objet au catalogue arbitraire d'une collection commerciale : "Pour ne pas s'empêtrer dans des définitions préalables, on se contentera d'une certitude de départ minimale : du roman western, c'est ce qu'a publié la collection du genre la plus durable en France, le “Masque Western", de 1967 à 1981 » (Bleton 14). Les romans d'Aimard, seuls titres français parus dans cette collection, en décalage d'un siècle et d'un continent avec le reste du catalogue, n'y devaient probablement leur présence qu'à la récente retombée des droits de leur auteur dans le domaine public.

3. Nos remarques elles-mêmes approfondissent une série de questions abordées dans plusieurs articles et travaux antérieurs (Di Gregorio, 2014; 2018).

4. Au-delà de ces concrétisations particulières, ce vocable de sauvagisme nous paraît pouvoir caractériser tout un imaginaire projeté sur l'espace « vacant » américain, et garanti par ce qu'on appellera, avec Fumaroli, la passion européenne de l'œil vierge. Au XIX $X^{\mathrm{e}}$ siècle, sauvagisme était presque un hapax. Il apparaît à plusieurs endroits de l'œuvre privée et publique de Victor Hugo, notamment dans une esquisse de l'humanité brossée dans William Shakespeare (1864). Mais c'est à un réformateur social, Charles Fourier, que le poète devait sa connaissance de ce terme. Dans sa Théorie de l'unité universelle (1822-23), Fourier définit précisément le sauvagisme comme un stade limbique garantissant à l'homme « les sept droits de nature, dont aucun ne peut lui être accordé en Civilisation » (41). Au-delà de l'acception idéologique, on devine ce qui, dans son sens et ses sonorités, a pu séduire Hugo : l'idée sous-jacente d'une sauvagerie heureuse et désirable, d'une mise en situation sauvage qui puisse être imitée, voire qui fasse système. C'est aussi en ce sens que nous l'employons pour tracer la continuité entre l'attrait pour le «sauvage ", omniprésent dans les projections françaises de l'Amérique, et le roman d'aventures que devait héroïser le coureur des bois.

5. Ce mythe de la Frontière est en effet celui que Buffalo Bill exhibera dans son Wild West Show (Di Gregorio 2015) et que le western entretiendra à Hollywood durant la plus grande partie du xx siècle. Extrapolé en historiographie par Frederick J. Turner à la fin du XIX siècle, ce mythe envisage l'Ouest américain comme l'espace d'une immense ligne de front en progression et dont tous les bénéfices, territoriaux comme culturels, seraient revenus aux États-Unis. C'est parce qu'un front de courageux pionniers aurait fait l'expérience du défrichage et du peuplement de tout un continent que les Américains, dans leur ensemble, seraient parvenus à forger leurs 
institutions propres et, finalement, leur identité nationale. La Frontière, dans cette perspective, ne représente pas un simple mouvement d'appropriation territoriale, mais bien un épiphanie culturelle permise par la conquête d'un immense espace vierge - ou présumé tel (Turner; Smith ; White \& Limerick). Pour une synthèse des débats autour de ce concept très discuté parmi les historiens et ethno-historiens, voir Villerbu 2014, 7-18.

6. Si l'appellation « coureur de bois » est historiquement la plus correcte, nous avons pris le parti, pour faire droit à la dimension romanesque du personnage, d'écrire, comme le faisaient Gabriel Ferry et Gustave Aimard, « coureur des bois » (cf. Havard) .

7. La participation organique du coureur aux grands bois majestueux qu'il arpente est rendue sensible dans l'iconographie fréquente qui accompagnait les romans sauvagistes, notamment la série de gravures réalisées par Gustave Doré pour Balle-Franche de Gustave Aimard (pré-originale dans le Journal pour tous, 1860).

8. Si Aimard, à l'instar de Ferry, élit souvent les États du Sud-Ouest dans ses romans, ces fameux borderlands très fréquentés par les voyageurs français avant et après la ruée vers l'or, il s'agit avant tout d'un "décor Potemkine" qui ne prend de sens que par le système d'aventure sauvagiste qui doit s'y déployer. D'ailleurs, même quand l'auteur les situe au Missouri, en Arkansas et au Canada, ses romans reproduisent rigoureusement la même mécanique aventureuse.

9. "One moccasin is no more like another than one book is like another; though they who can read in one are seldom able to tell the marks of the other. " (Cooper, 1826, chapter XVIII)

10. " "Uncas was bold enough to say, that the beasts ridden by the gentle ones," continued Hawkeye, glancing his eyes, not without curious interest, on the fillies of the ladies, "planted the legs of one side on the ground at the same time, which is contrary to the movements of all trotting four-footed animals of my knowledge, except the bear. And yet here are horses that always journey in this manner, as my own eyes have seen, and as their trail has shown for twenty long miles. [...] But, go sideling or go straight, Uncas had seen the movement, and their trail led us on to the broken bush. The outer branch, near the prints of one of the horses, was bent upward, as a lady breaks a flower from its stem, but all the rest were ragged and broken down, as if the strong hand of a man had been tearing them! So I concluded that the cunning varmints had seen the twig bent, and had torn the rest, to make us believe a buck had been feeling the boughs with his antlers." » (Cooper, 1826, chapter XVIII)

\section{RÉSUMÉS}

Cet article interroge l'imaginaire américain déployé dans le roman sauvagiste français : porté notamment par Gustave Aimard (1818-1883) et son énorme production, ce sous-genre du feuilleton d'aventures fut l'un des plus répandus entre 1850 et 1880 . L'analyse se penche principalement sur l'héroïsme spécifique incarné par le coureur des bois, héros récurrent de ces romans, mais aussi sur les modalités d'interaction et d'évolution de ce personnage dans l'Ouest romanesque. Dans une perspective d'histoire culturelle et de littérature comparée, ce romanesque du trappeur sera mis en contraste avec le langage classique du western (déchiffrage vs. défrichage, marche vs. cheval, lutte des traces vs. effraction et conquête) afin d'insister sur la valeur d'alternative imaginaire qu'a représentée ce sauvagisme aventureux (et largement 
européen) face à l'aventure du pionnier, nourrie, à la même époque, par les discours de la Destinée Manifeste et de la Frontière.

This paper analyzes the representation of North-American fictional worlds in the French western novel. With writers such as Gustave Aimard (1818-1883), the subgenre was one of the most common adventure serials between 1850 and 1880 . The analysis deals with the heroism embodied by the recurring figure of the coureur des bois, a French variety of mountain man who is the main protagonist of these novels. I question the relationship between that character and the fictional West. Through a historical and comparative approach, the coureurs des bois' adventure will be compared with the classical language of the Western (deciphering vs. settlement, walking vs. mounting, tracking vs. invasion and conquest) so as to insist on the alternative value brought by the adventurous "sauvagisme" (mainly European) in contrast with pioneers/settlers stories which were inspired, during the same period, by the rhetorics of the manifest destiny and the frontier.

INDEX

Keywords : trapper, mountain man, frontier, American West, western, clues, American Indian (myth), savagery, Gustave Aimard

Mots-clés : trappeur, coureur des bois, Frontière, Ouest américain, western, paradigme indiciaire, Bon Sauvage, sauvagisme, Gustave Aimard

\section{AUTEUR}

\section{LUCA DI GREGORIO}

Université de Liège

ldigregorio@uliege.be 